Mathematical Research Letters 8, 429-435 (2001)

\title{
HYPERELLIPTIC JACOBIANS WITHOUT COMPLEX MULTIPLICATION IN POSITIVE CHARACTERISTIC
}

\author{
YURI G. ZARHIN
}

\section{Introduction}

The aim of this note is to prove that in positive characteristic $p \neq 2$ the jacobian $J(C)=J\left(C_{f}\right)$ of a hyperelliptic curve

$$
C=C_{f}: y^{2}=f(x)
$$

has only trivial endomorphisms over an algebraic closure $K_{a}$ of the ground field $K$ if the Galois group $\operatorname{Gal}(f)$ of the polynomial $f \in K[x]$ of even degree is "very big".

More precisely, if $f$ is a polynomial of even degree $n \geq 10$ and $\operatorname{Gal}(f)$ is either the symmetric group $\mathbf{S}_{n}$ or the alternating group $\mathbf{A}_{n}$ then $\operatorname{End}(J(C))=\mathbf{Z}$. Notice that it is known [14] that in this case (and even for all integers $n \geq 5$ ) either $\operatorname{End}(J(C))=\mathbf{Z}$ or $J(C)$ is a supersingular abelian variety and the real problem is how to prove that $J(C)$ is not supersingular.

There are some results of this type in the literature. Previously Mori [7], [8] has constructed explicit examples of hyperelliptic jacobians without nontrivial endomorphisms. Namely, he proved that if $K=k(z)$ is a field of rational functions in variable $z$ with constant field $k$ of characteristic $p \neq 2$ then for each integer $g \geq 2$ the $g$-dimensional jacobian of a hyperelliptic $K$-curve

$$
y^{2}=x^{2 g+1}-x+z
$$

has no nontrivial endomorphisms over $K_{a}$ if $p$ does not divide $g(2 g+1)$.

I am deeply grateful to the referee for helpful suggestions.

\section{Main result}

Throughout this paper we assume that $K$ is a field of prime characteristic $p$ different from 2. We fix its algebraic closure $K_{a}$ and write $\operatorname{Gal}(K)$ for the absolute Galois group $\operatorname{Aut}\left(K_{a} / K\right)$.

Theorem 2.1. Let $K$ be a field with $p=\operatorname{char}(K)>2, K_{a}$ its algebraic closure, $f(x) \in K[x]$ an irreducible separable polynomial of even degree $n \geq 10$ such that the Galois group of $f$ is either $\mathbf{S}_{n}$ or $\mathbf{A}_{n}$. Let $C_{f}$ be the hyperelliptic curve

Received February 2, 2001.

Partially supported by the NSF. 
$y^{2}=f(x)$. Let $J\left(C_{f}\right)$ be its jacobian, $\operatorname{End}\left(J\left(C_{f}\right)\right)$ the ring of $K_{a}$-endomorphisms of $J\left(C_{f}\right)$. Then $\operatorname{End}\left(J\left(C_{f}\right)\right)=\mathbf{Z}$.

Examples 2.2. Let $k$ be a field of odd prime characteristic $p$. Let $k(z)$ be the field of rational functions in variable $z$ with constant field $k$. We write $\overline{k(z)}$ for an algebraic closure of $k(z)$.

(i) Suppose $K_{n}=k\left(z_{1}, \cdots, z_{n}\right)$ is the field of rational functions in $n$ independent variables $z_{1}, \cdots, z_{n}$ over $k$. Then the Galois group of a polynomial $x^{n}-z_{1} x^{n-1}+\cdots+(-1)^{n} z_{n}$ over $K_{n}$ is $\mathbf{S}_{n}$. Therefore if $n \geq 10$ is even then the jacobian of the curve $y^{2}=x^{n}-z_{1} x^{n-1}+\cdots+(-1)^{n} z_{n}$ has no nontrivial endomorphisms over an algebraic closure of $K_{n}$.

(ii) Suppose $p$ does not divide $n$ and $h(x) \in k[x]$ is a Morse polynomial of degree $n$. This means that the derivative $h^{\prime}(x)$ of $h(x)$ has $n-1$ distinct roots $\beta_{1}, \cdots \beta_{n-1}$ (in an algebraic closure of $k$ ) and $h\left(\beta_{i}\right) \neq h\left(\beta_{j}\right)$ while $i \neq j$. For example, $h(x)=x^{n}-x$ enjoys these properties if and only if $p$ does not divide $n(n-1)$.

Then the Galois group of $h(x)-z$ over $k(z)$ is $\mathbf{S}_{n}$ ([10], Th. 4.4.5, p. 41). Hence if $n \geq 10$ is even then the jacobian of the curve $y^{2}=h(x)-z$ has no nontrivial endomorphisms over $\overline{k(z)}$. In particular, for each integer $g \geq 4$ the $g$-dimensional jacobian of a hyperelliptic $K$-curve $y^{2}=x^{2 g+2}-x-z$ has no nontrivial endomorphisms over $\overline{k(z)}$ if $p$ does not divide $(g+1)(2 g+1)$.

(iii) Suppose $k$ is algebraically closed. Suppose an integer $q>1$ is a power of $p$ and $t$ is a positive integer not divisible by $p$. Let us choose a positive integer $s$ and a non-zero element $a$ of $k$.

(a) Assume that $t>q$ and let us put $n=q+t$. The Galois group of $x^{n}-z x^{t}+1$ over $k(z)$ is $\mathbf{A}_{n}$ ([1], Th. 1, p. 67). Clearly, if $t$ is odd then $n=q+t$ is even and $n>2 q \geq 6$, i.e., $n \geq 8$. In addition, $n \geq 10$ unless $q=3, t=5$. This implies that if $t$ is odd and $(q, t) \neq(3,5)$ then the jacobian of the curve $y^{2}=x^{n}-z x^{t}+1$ has no nontrivial endomorphisms over $\overline{k(z)}$.

(b) Assume that $n=2 p d \geq 10$ for some positive integer $d$ and $1<t<p d$. Assume, in addition that $t$ and $n$ are relatively prime and $s$ is divisible by $t$ (e.g., $t=s=p d-1$ if $d$ is even). The Galois group of $x^{n}-a x^{t}+z^{s}$ over $k(z)$ is $\mathbf{A}_{n}$ ([2], p. 107). Therefore the jacobian of the hyperelliptic curve $y^{2}=x^{n}-a x^{t}+z^{s}$ has no nontrivial endomorphisms over $\overline{k(z)}$.

As was already pointed out, in light of Th. 2.1 of [14], our Theorem 2.1 is an immediate corollary of the following auxiliary statement.

Theorem 2.3. Suppose $n=2 g+2$ is an even integer which is greater than or equal to 10. Suppose $f(x) \in K[x]$ is a separable polynomial of degree $n$, whose Galois group is either $\mathbf{A}_{n}$ or $\mathbf{S}_{n}$. Suppose $C$ is the hyperelliptic curve $y^{2}=f(x)$ of genus $g$ over $K$ and $J(C)$ is the jacobian of $C$.

Then $J(C)$ is not a supersingular abelian variety. 
Remark 2.4. Replacing (in the case of $\operatorname{Gal}(f)=\mathbf{S}_{n}$ ) $K$ by its proper quadratic extension, we may assume in the course of the proof of Theorem 2.3 that $\operatorname{Gal}(f)=\mathbf{A}_{n}$. Also, replacing $K$ by its abelian extension obtained by adjoining to $K$ all 2-power roots of unity, we may assume that $K$ contains all 2-power roots of unity.

We prove Theorem 2.3 in the next Section.

\section{Proof of Theorem 2.3}

So, we assume that $K$ contains all 2-power roots of unity, $f(x) \in K[x]$ is an irreducible separable polynomial of even degree $n=2 g+2 \geq 10$ and $\operatorname{Gal}(f)=$ $\mathbf{A}_{n}$. Therefore $J(C)$ is a $g$-dimensional abelian variety defined over $K$. The group $J(C)_{2}$ of its points of order 2 is a $2 g$-dimensional $\mathbf{F}_{2}$-vector space provided with the natural action of $\mathrm{Gal}(K)$. It is well-known (see for instance [15], Sect. $5)$ that the image of $\operatorname{Gal}(K)$ in $\operatorname{Aut}\left(J(C)_{2}\right)$ is canonically isomorphic to $\operatorname{Gal}(f)$.

Now Theorem 2.3 becomes an immediate corollary of the following two assertions.

Lemma 3.1. Let $F$ be a field, whose characteristic is not 2 and assume that $F$ contains all 2-power roots of unity. Let $g$ be a positive integer and $G$ be a finite simple non-abelian group enjoying the following properties:

(a) Each nontrivial representation of $G$ in characteristic 0 has dimension $>2 g$;

(b) If $G^{\prime} \rightarrow G$ is a surjective group homomorphism, whose kernel is a central subgroup of order 2 then each faithful absolutely irreducible representation of $G^{\prime}$ in characteristic zero has dimension $\neq 2 g$.

(c) Each nontrivial representation of $G$ in characteristic 2 has dimension $\geq 2 g$.

If $X$ is a $g$-dimensional abelian variety over $F$ such that the image of $\operatorname{Gal}(F)$ in $\operatorname{Aut}\left(X_{2}\right)$ is isomorphic to $G$ then $X$ is not supersingular.

In order to state the second assertion we need to recall the following definition ([13], p. 584). If $V$ is a finite-dimensional vector space over an algebraically closed field then a projective representation $\rho: G \rightarrow \operatorname{PGL}(V)$ is called proper if there is no a linear representation $\rho^{\prime}: G \rightarrow \operatorname{GL}(V)$ such that $\rho=\pi \rho^{\prime}$ where $\pi: \mathrm{GL}(V) \rightarrow \mathrm{PGL}(V)$ is the natural surjection.

Lemma 3.2. Suppose $n=2 g+2 \geq 10$ is an even integer. Let us put $G=\mathbf{A}_{n}$. Then:

(a) Each nontrivial representation of $G$ in characteristic 0 has dimension $\geq$ $n-1>2 g$

(b) Each proper projective representation of $G$ in characteristic 0 has dimension $\neq 2 g$

(c) Each nontrivial representation of $G$ in characteristic 2 has dimension $\geq 2 g$.

Lemma 3.1 will be proven in the next Section. We prove Lemma 3.2 in Section 5 . 


\section{Not supersingularity}

We keep all the notations of Lemma 3.1. Assume that $X$ is supersingular. Our goal is to get a contradiction. We write $T_{2}(X)$ for the 2 -adic Tate module of $X$ and

$$
\rho_{2, X}: \operatorname{Gal}(F) \rightarrow \operatorname{Aut}_{\mathbf{Z}_{2}}\left(T_{2}(X)\right)
$$

for the corresponding 2-adic representation. It is well-known that $T_{2}(X)$ is a free $\mathbf{Z}_{2}$-module of $\operatorname{rank} 2 \operatorname{dim}(X)=2 g$ and

$$
X_{2}=T_{2}(X) / 2 T_{2}(X)
$$

(the equality of Galois modules). Let us put

$$
H=\rho_{2, X}(\operatorname{Gal}(F)) \subset \operatorname{Aut}_{\mathbf{Z}_{2}}\left(T_{2}(X)\right) .
$$

Clearly, the natural homomorphism

$$
\bar{\rho}_{2, X}: \operatorname{Gal}(F) \rightarrow \operatorname{Aut}\left(X_{2}\right)
$$

defining the Galois action on the points of order 2 is the composition of $\rho_{2, X}$ and (surjective) reduction map modulo 2

$$
\operatorname{Aut}_{\mathbf{Z}_{2}}\left(T_{2}(X)\right) \rightarrow \operatorname{Aut}\left(X_{2}\right) .
$$

This gives us a natural (continuous) surjection

$$
\pi: H \rightarrow \bar{\rho}_{2, X}(\operatorname{Gal}(F)) \cong G,
$$

whose kernel consists of elements of $1+2 \operatorname{End}_{\mathbf{z}_{2}}\left(T_{2}(X)\right)$. It follows from the property $3.1(\mathrm{c})$ and equality $\operatorname{dim}_{\mathbf{F}_{2}}\left(X_{2}\right)=2 g$ that the $G$-module $X_{2}$ is absolutely simple and therefore the $H$-module $X_{2}$ is also absolutely simple. Here the structure of $H$-module is defined on $X_{2}$ via

$$
H \subset \operatorname{Aut}_{\mathbf{z}_{2}}\left(T_{2}(X)\right) \rightarrow \operatorname{Aut}\left(X_{2}\right) .
$$

The absolute simplicity of the $H$-module $X_{2}$ means that the natural homomorphism

$$
\mathbf{F}_{2}[H] \rightarrow \operatorname{End}_{\mathbf{F}_{2}}\left(X_{2}\right)
$$

is surjective ([4], Th. 9.2 on p. 145). By Nakayama's Lemma, this implies that another natural homomorphism

$$
\mathbf{Z}_{2}[H] \rightarrow \operatorname{End}_{\mathbf{Z}_{2}}\left(T_{2}(X)\right)
$$

is also surjective (see [6], p. 252).

Let $V_{2}(X)=T_{2}(X) \otimes \mathbf{z}_{2} \mathbf{Q}_{2}$ be the $\mathbf{Q}_{2}$-Tate module of $X$. It is well-known that $V_{2}(X)$ is the $2 g$-dimensional $\mathbf{Q}_{2}$-vector space and $T_{2}(X)$ is a $\mathbf{Z}_{2}$-lattice in $V_{2}(X)$. Clearly, the $\mathbf{Q}_{2}[H]$-module $V_{2}(X)$ is also absolutely simple.

The choice of polarization on $X$ gives rise to a non-degenerate alternating bilinear form (Riemann form) [9]

$$
e: V_{2}(X) \times V_{2}(X) \rightarrow \mathbf{Q}_{2}(1) \cong \mathbf{Q}_{2}
$$


Since $F$ contains all 2-power roots of unity, $e$ is $\operatorname{Gal}(F)$-invariant and therefore is $H$-invariant. In particular,

$$
H \subset \mathrm{SL}\left(V_{2}(X)\right)
$$

There exists a finite Galois extension $L$ of $F$ such that all endomorphisms of $X$ are defined over $L$. We write $\operatorname{End}^{0}(X)$ for the $\mathbf{Q}$-algebra $\operatorname{End}(X) \otimes \mathbf{Q}$ of endomorphisms of $X$. Since $X$ is supersingular,

$$
\operatorname{dim}_{\mathbf{Q}} \operatorname{End}^{0}(X)=(2 \operatorname{dim}(X))^{2}=(2 g)^{2} .
$$

Recall ([9]) that the natural map

$$
\operatorname{End}^{0}(X) \otimes_{\mathbf{Q}} \mathbf{Q}_{2} \rightarrow \operatorname{End}_{\mathbf{Q}_{2}} V_{2}(X)
$$

is an embedding. Dimension arguments imply that

$$
\operatorname{End}^{0}(X) \otimes_{\mathbf{Q}} \mathbf{Q}_{2}=\operatorname{End}_{\mathbf{Q}_{2}} V_{2}(X) .
$$

Since all endomorphisms of $X$ are defined over $L$, the image

$$
\rho_{2, X}(\operatorname{Gal}(L)) \subset \rho_{2, X}(\operatorname{Gal}(F)) \subset \operatorname{Aut}_{\mathbf{Z}_{2}}\left(T_{2}(X)\right) \subset \operatorname{Aut}_{\mathbf{Q}_{2}}\left(V_{2}(X)\right)
$$

commutes with $\operatorname{End}^{0}(X)$. This implies that $\rho_{2, X}(\operatorname{Gal}(L))$ commutes with $\operatorname{End}_{\mathbf{Q}_{2}} V_{2}(X)$ and therefore consists of scalars. Since

$$
\rho_{2, X}(\operatorname{Gal}(L)) \subset \rho_{2, X}(\operatorname{Gal}(F)) \subset \operatorname{SL}\left(V_{2}(X)\right),
$$

$\rho_{2, X}(\operatorname{Gal}(L))$ is a finite group. Since $\operatorname{Gal}(L)$ is a subgroup of finite index in $\operatorname{Gal}(F)$, the group $H=\rho_{2, X}(\operatorname{Gal}(F))$ is also finite. In particular, the kernel of the reduction map modulo 2

$$
\operatorname{Aut}_{\mathbf{Z}_{2}} T_{2}(X) \supset H \rightarrow G \subset \operatorname{Aut}\left(X_{2}\right)
$$

consists of periodic elements and, thanks to Minkowski-Serre Lemma [11], $Z:=\operatorname{ker}(H \rightarrow G)$ has exponent 1 or 2 . In particular, $Z$ is commutative. Since

$$
Z \subset H \subset \mathrm{SL}\left(V_{2}(X)\right),
$$

$Z$ is a $\mathbf{F}_{2}$-vector space of dimension $d<2 g$. This implies that the adjoint action

$$
H \rightarrow H / Z=G \rightarrow \operatorname{Aut}(Z) \cong \mathrm{GL}_{d}\left(\mathbf{F}_{2}\right)
$$

is trivial, in light of property 3.1(c). This means that $Z$ lies in the center of $H$. Since the $\mathbf{Q}_{2}[H]$-module $V_{2}(X)$ is faithful absolutely simple, $Z$ consists of scalars. This implies that either $Z=\{1\}$ or $Z=\{ \pm 1\}$. If $Z=\{1\}$ then $H \cong G$ and $V_{2}(X)$ is a faithful $\mathbf{Q}_{2}[G]$-module of dimension $2 g$ which contradicts the property 3.1(a). Therefore $Z=\{ \pm 1\}$ and $H \rightarrow G$ is a surjective group homomorphism, whose kernel is a central subgroup of order 2. But $V_{2}(X)$ is a faithful absolutely simple $\mathbf{Q}_{2}[H]$-module of dimension $2 g$ which contradicts the property 3.1(b). This ends the proof of Lemma 3.1. 


\section{Representation theory}

Proof of Lemma 3.2. The property (a) follows easily from Th. 2.5.15 on p. 71 of [5]. The property (c) follows readily from Th. 1.1 on p. 127 of [12]. The rest of this Section is devoted to the proof of the property (b). First, notice that the case $n=10$ follows from Tables in [3]. So, further we assume that $n \geq 12$.

We start with an elementary discussion of the dyadic expansion $n=2^{w_{1}}+$ $\cdots+2^{w_{s}}$ of $n$. Here $w_{i}$ 's are distinct nonnegative integers with $w_{1}<\cdots<w_{s}$ and $s$ is the exact number of terms (non-zero digits) in the dyadic expansion of $n$. Since $n$ is even, $w_{1} \geq 1$ and therefore each $w_{i} \geq i$. This implies that $n \geq 2\left(2^{s}-1\right)=2^{s+1}-2$.

By a theorem of Wagner (Th. 1.3(ii) on pp. 583-584 of [13]), each proper projective representation of $\mathbf{A}_{n}$ in characteristic $\neq 2$ has dimension divisible by $N:=2^{\left\lfloor\frac{n-s-1}{2}\right\rfloor}$. So, in order to prove (b), it suffices to check that $n-2$ is not divisible by $N$ for all even $n \geq 12$.

If $n=12$, it is verified immediately. If $n \geq 14$ then $2^{n-2}>(n+1)(n-2)^{2}$. Then $2^{n-\log _{2}(n+1)-2}>(n-2)^{2}$. It is easy to see that $s \leq \log _{2}(n+1)$, so $2^{n-s-2}>(n-2)^{2}$. Taking square roots at both sides, we get $2^{\frac{n-s-2}{2}}>n-2$. Then we see easily that $2^{\left\lfloor\frac{n-s-1}{2}\right\rfloor}>n-2$. This finishes the proof of (b).

\section{Corrigendum to $[15]$}

Page 475, Remarks 2.2, last line: read "absolutely simple" instead of "also very simple".

Page 478, line -5: read " $\operatorname{Gal}(K)$ " instead of " $G(K)$ ".

\section{References}

[1] S. S. Abhyankar, Alternating group coverings of the affine line for characteristic greater than two, Math. Ann. 296 (1993), 63-68.

[2] _ Galois theory on the line in nonzero characteristic, Bull. Amer. Math. Soc. 27 (1992), 68-133.

[3] J. H. Conway, R. T. Curtis, S. P. Norton, R. A. Parker, and R. A. Wilson, Atlas of finite groups, Oxford University Press, Oxford, 1985.

[4] I. M. Isaacs, Character theory of finite groups, Pure and Applied Mathematics, No. 69, Academic Press, New York-San Francisco-London, 1976.

[5] G. James and A. Kerber, The representation theory of the symmetric group, Addison Wesley Publishing Company, Reading, MA, 1981.

[6] B. Mazur, Deformation theory of Galois representations, Modular forms and Fermat's last theorem (G. Cornell, J. H. Silverman, G. Stevens, eds.), Springer-Verlag, New York, 1997, pp. 243-311.

[7] Sh. Mori, The endomorphism rings of some abelian varieties, Japan. J. Math. 2 (1976), 109-130.

[8] _ The endomorphism rings of some abelian varieties II, Japan. J. Math. 3 (1977), 105-109.

[9] D. Mumford, Abelian varieties, second edition, Oxford University Press, London, 1974.

[10] J.-P. Serre, Topics in Galois Theory, Res. Notes Math. 1, Jones and Bartlett Publishers, Boston-London, 1992. 
[11] A. Silverberg and Y. G. Zarhin, Variations on a theme of Minkowski and Serre, J. Pure Appl. Algebra 111 (1996), 285-302.

[12] A. Wagner, The faithful linear representations of $\mathbf{S}_{n}$ and $\mathbf{A}_{n}$ over a field of characteristic 2, Math. Z. 151 (1976), 127-137.

[13] An observation on the degrees of the symmetric and alternating group over an arbitrary field, Arch. Math. 29 (1977), 583-589.

[14] Y. G. Zarhin, Hyperelliptic jacobians without complex multiplication, Math. Res. Lett. 7 (2000), 123-132.

[15] Hyperelliptic jacobians and modular representations, Moduli of abelian varieties (eds. G. van der Geer, C. Faber, F. Oort), Progr. Math. 195, Birkhäuser, Basel-BostonBerlin, 2001, pp. 473-490.

Dept. of Mathematics, Pennsylvania State University, University Park, PA 16802. Institute for Mathematical Problems in Biology, Russian Academy of Sciences, Pushchino, Moscow Region, 142292, RUSSIA.

E-mail address: zarhin@math.psu.edu 